\title{
Effects of Tocilizumab Therapy on Serum Interleukin-33 and Interleukin-6 Levels in Patients With Rheumatoid Arthritis
}

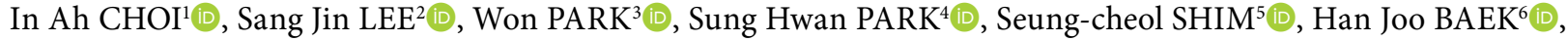

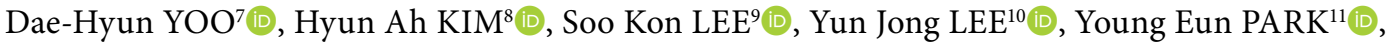 \\ Hoon-Suk CHA ${ }^{12}$ D , Eun Young LEE ${ }^{13}$ D, Eun Bong LEE $^{13}$ D, Yeong Wook SONG ${ }^{2}$ \\ ${ }^{1}$ Department of Internal Medicine, Chungbuk National University Hospital, Cheongju, South Korea \\ ${ }^{2}$ Department of Molecular Medicine and Biopharmaceutical Sciences, Graduate School of Convergence Science and Technology, \\ and College of Medicine, Medical Research Institute, Seoul National University, Seoul, South Korea \\ ${ }^{3}$ Department of Internal Medicine, Inha University Hospital, Incheon, South Korea \\ ${ }^{4}$ Department of Internal Medicine, The Catholic University of Korea Seoul St. Mary's Hospital, Seoul, South Korea \\ ${ }^{5}$ Department of Internal Medicine, Chungnam National University Hospital, Daejeon, South Korea \\ ${ }^{6}$ Department of Internal Medicine, Gachon University Gil Medical Center, Incheon, South Korea \\ ${ }^{7}$ Department of Internal Medicine, Hanyang University Medical Center, Seoul, South Korea \\ ${ }^{8}$ Department of Internal Medicine, Hallym University Medical Center, Anyang, South Korea \\ ${ }^{9}$ Department of Internal Medicine, CHA Bundang Medical Center, Seongnam, South Korea \\ ${ }^{10}$ Department of Internal Medicine, Seoul National University Bundang Hospital, Seongnam, South Korea \\ ${ }^{11}$ Department of Internal Medicine, Pusan National University Hospital, Busan, South Korea \\ ${ }^{12}$ Department of Internal Medicine, Sam Sung Medical Center, Seoul, Seoul, South Korea \\ ${ }^{13}$ Department of Internal Medicine, Seoul National University Hospital, Seoul, South Korea
}

\begin{abstract}
Objectives: This study aims to examine the effects of tocilizumab therapy on serum levels of interleukin (IL)-33 and IL- 6 in rheumatoid arthritis (RA) patients.

Patients and methods: Interleukin-33 and IL- 6 levels in serum samples from 83 RA patients (10 males, 73 females; mean age 51.9 years; range, 26 to 77 years) and 12 healthy controls ( 2 males, 10 females; mean age 52.2 years; range, 39 to 62 years) were compared by cross-sectional, case control analysis. Of the RA patients, 40 received 24 weeks of tocilizumab therapy and were assigned to the prospective cohort. These 40 patients were then divided into two subgroups as responders and non-responders according to the American College of Rheumatology (ACR) 20. The remaining 43 RA patients did not receive tocilizumab therapy. Serum cytokine levels were analyzed at baseline and after 24 weeks of tocilizumab therapy in these patients.

Results: Interleukin-33 and IL- 6 concentrations were significantly higher in RA patients than in healthy controls ( $p<0.001$ for both). Serum IL-33 levels in RA patients showed a significant correlation with rheumatoid factor titer $(r=0.660, p<0.001)$, and IL- 6 levels showed a significant correlation with high-sensitivity C-reactive protein levels (Spearman's rank correlation coefficient $=0.482, p<0.001$ ). Serum IL-33 levels decreased significantly after 24 weeks of tocilizumab therapy $(p<0.001)$; this was particularly marked in ACR20 responders $(p<0.001)$. However, the decrease in non-responders was not significant $(p=0.066)$. Changes in serum IL-6 levels after 24 weeks of tocilizumab therapy were not significant in either ACR20 responders or non-responders.
\end{abstract}

Conclusion: Serum IL-33 levels in RA patients receiving tocilizumab therapy decreased significantly, particularly in ACR20 responders. Thus, IL-33 may be a useful marker for monitoring responses to tocilizumab therapy.

Keywords: Arthritis; interleukin-6; interleukin-33; rheumatoid; tocilizumab.

Received: November 23, 2016 Accepted: January 22, 2018 Published online: March 23, 2018

Correspondence: Yeong Wook Song, MD. Department of Molecular Medicine and Biopharmaceutical Sciences, Graduate School of Convergence Science and Technology, and College of Medicine, Medical Research Institute, Seoul National University, 03080 Seoul, South Korea.

Tel: +82-10-3796-4135 e-mail: ysong@snu.ac.kr

Choi IA, Lee SJ, Park W, Park SH, Shim SC, Baek HJ. Effects of tocilizumab therapy on serum interleukin-33 and interleukin- 6 levels in patients with rheumatoid arthritis. Arch Rheumatol 2018;33(4):389-394. 
Rheumatoid arthritis (RA) is a chronic inflammatory disease of the synovial joints, which results in cartilage and bone destruction. Several pro-inflammatory cytokines such as tumor necrosis factor-alpha (TNF)- $\alpha$, interleukin (IL)-1, IL-6, IL-8, and IL-15 are critical for synovial inflammation in RA, ${ }^{1,2}$ indeed, treating RA by targeting pro-inflammatory cytokines, including TNF- $\alpha$, IL-1, and IL-6, has met with some success. ${ }^{3-5}$

Interleukin-33 is a newly described member of the IL-1 family and was identified as a ligand for the orphan receptor growth STimulation expressed gene 2 (ST 2). It plays a role in allergic diseases by activating mast cells and promotes T helper type 2 immune responses. ${ }^{6,7}$ IL-33 levels in RA patients are higher than those in healthy controls, and synovial expression of IL-33 is greater in RA than in osteoarthritis, suggesting that IL-33 may be involved in the pathogenesis of RA. ${ }^{8,9}$ In addition, blocking IL-33 ameliorates joint inflammation and destruction in mice with collagen-induced arthritis (CIA)..$^{10,11}$

A previous study has shown that serum levels of IL-33 correlate with those of IL-6 in RA patients. ${ }^{8}$ However, to our knowledge, no study has examined serum IL-33 levels in RA patients receiving IL-6 receptor (IL-6R) inhibitor therapy. Therefore, in this study, we aimed to examine the effects of tocilizumab therapy on serum levels of IL-33 and IL-6 in RA patients.

\section{PATIENTS AND METHODS}

We performed a cross-sectional, case control analysis of serum IL-33 and IL-6 levels in 83 patients with RA (10 males, 73 females; mean age 51.9 years; range, 26 to 77 years) and 12 healthy controls ( 2 males, 10 females; mean age 52.2 years; range, 39 to 62 years) at participating hospitals between September 2009 and December 2010. Of the RA patients, 40 were treated with an intravenous infusion of $8 \mathrm{mg} / \mathrm{kg}$ tocilizumab every four weeks for 24 weeks with a stable dose of methotrexate and/or other diseasemodifying anti-rheumatic drugs. Therefore, these 40 RA patients were considered the prospective cohort. The 40 patients were then divided into two subgroups as responders and nonresponders according to the American College of

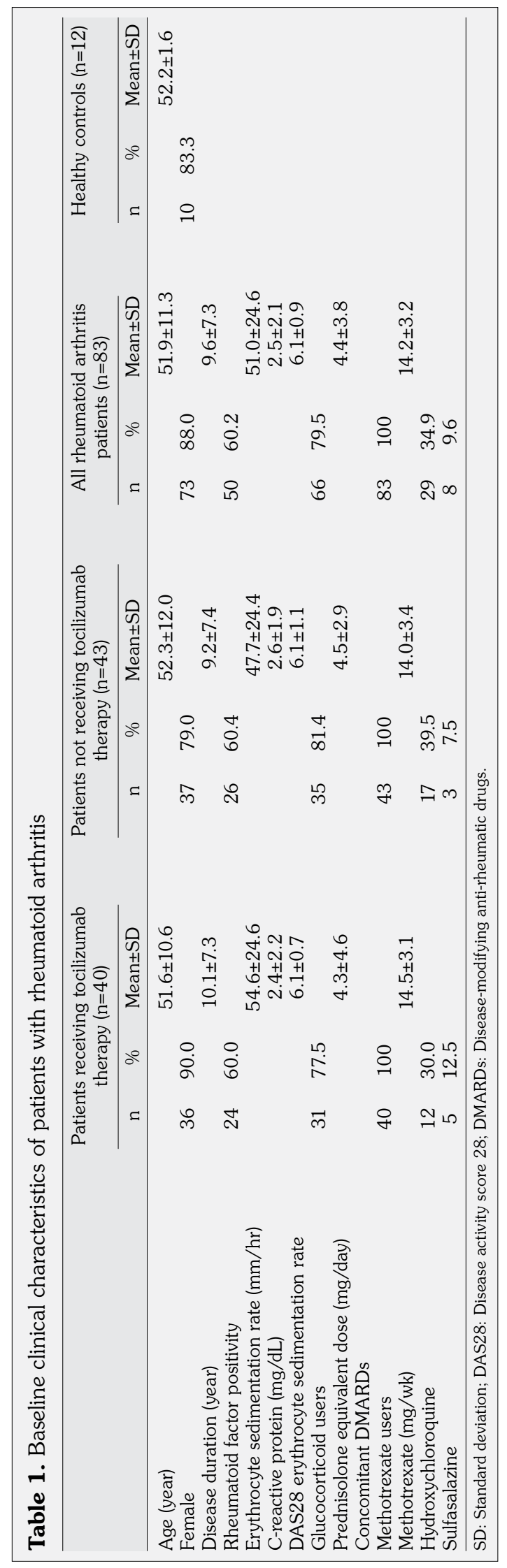


(a)

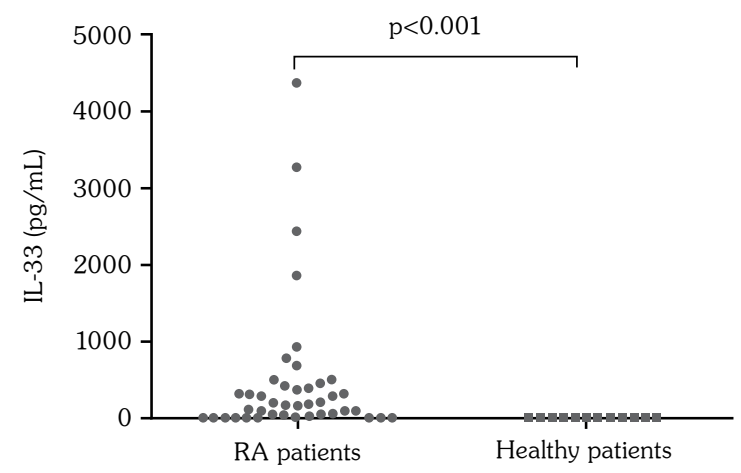

(b)

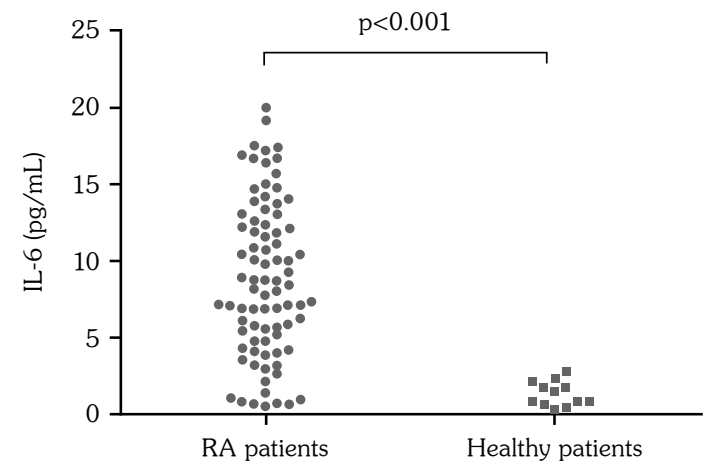

Figure 1. Serum interleukin-33 and interleukin- 6 concentrations in 83 patients with rheumatoid arthritis and 12 healthy controls. Serum interleukin-33 (a) and interleukin-6 concentrations (b) were significantly higher in patients with rheumatoid arthritis than in healthy controls. Bars represent median values. IL: Interleukin; RA: Rheumatoid arthritis.

Rheumatology (ACR) 20. The remaining $43 \mathrm{RA}$ patients did not receive tocilizumab therapy.The study protocol was approved by the Seoul National University Hospital Ethics Committee (C-1302-028-463). A written informed consent was obtained from each patient. The study was conducted in accordance with the principles of the Declaration of Helsinki.

Serum samples were promptly stored at $-80^{\circ} \mathrm{C}$ until the time of cytokine assay. Samples from the 40 patients that received tocilizumab were obtained at two time points: at baseline and 24 weeks after the initial dose was given. Clinical scores and laboratory assay results, including the erythrocyte sedimentation rate (ESR), highsensitivity C-reactive protein (hsCRP) level, serum rheumatoid factor (RF) level, and disease activity score of 28 joints including the ESR (DAS28-ESR), were obtained at the same time points.

Interleukin-33 was measured using a bead immunoassay and Luminex laser-based fluorescent analytical test instrumentation (Merck-Millipore, Merck KGaA, Darmstadt, Germany). Serum IL-6 and RF were assayed using enzyme linked immunosorbent assay (ELISA) (IL-6, eBioscience, San Diego, CA, USA and RF, Nittobo, Tokyo, Japan). All assays were conducted in duplicate.

\section{Statistical analysis}

Data are expressed as the mean \pm standard deviation or median (range) as appropriate. The Mann-Whitney $\mathrm{U}$ test was used for group (a)

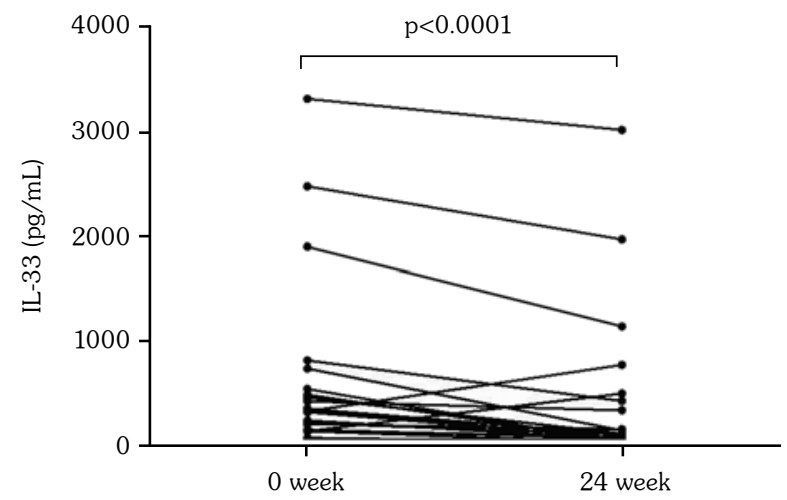

(b)

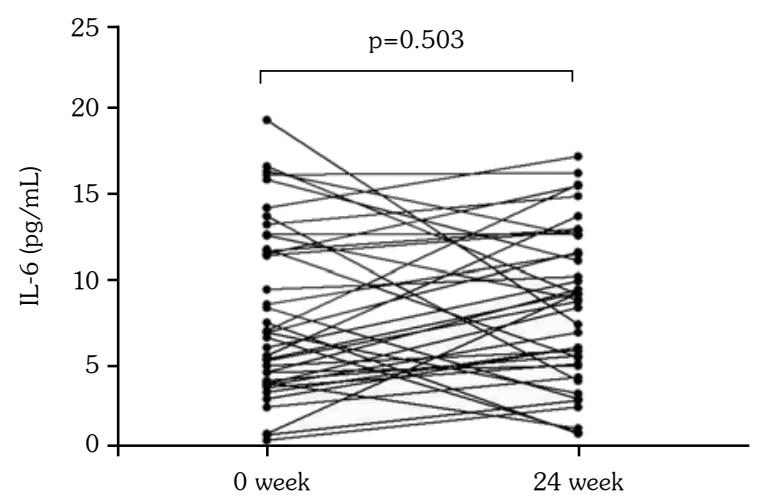

Figure 2. Serum interleukin-33 and interleukin-6 concentrations in patients with rheumatoid arthritis before and after 24 weeks of tocilizumab therapy $(n=40)$. (a) Serum interleukin-33 concentrations were significantly lower after tocilizumab therapy, (b) but serum interleukin-6 concentrations were not. IL: Interleukin. 
(a)

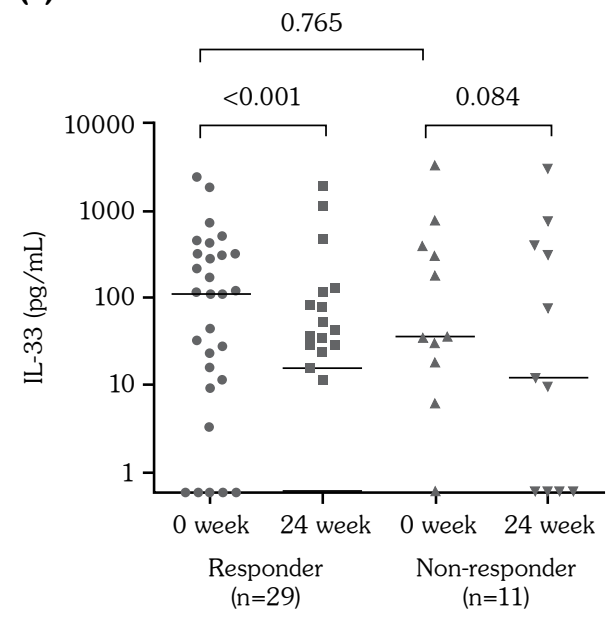

(b)

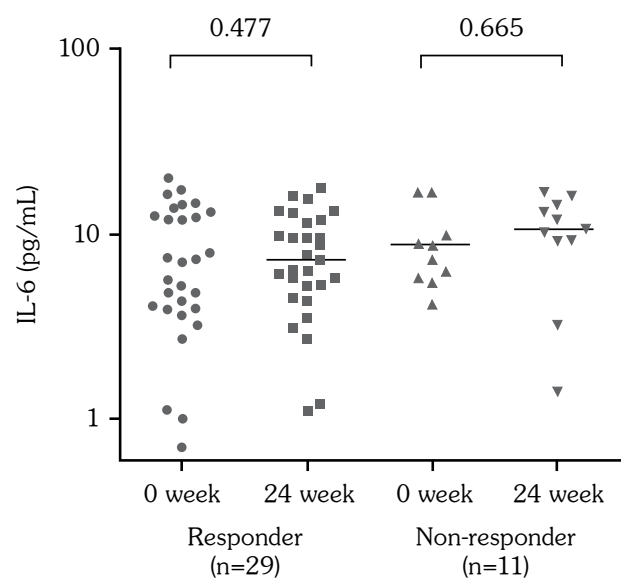

Figure 3. Serum interleukin-33 and interleukin- 6 concentrations in American College of Rheumatology 20 responders and non-responders before and after 24 weeks of tocilizumab therapy. (a) Serum interleukin-33 levels in American College of Rheumatology 20 responders decreased significantly following tocilizumab therapy; this was not the case for the non-responder group. Baseline interleukin-33 levels in these two groups were not significantly different. (b) Changes in interleukin- 6 levels in both groups after 24 weeks of tocilizumab therapy were not significant. Bars represent median values. IL: Interleukin.

comparisons and the Wilcoxon signed rank test for paired comparisons. Single regression analysis was performed to examine the relationship between serum IL-33 levels and clinical laboratory parameters, and Spearman's rank correlation coefficients were calculated. The Wilcoxon signed rank test was used to compare values measured before and after tocilizumab therapy. $P$ values $<0.05$ were considered significant. All statistical analyses were performed using IBM SPSS statistics version 22.0 (IBM Corp., Armonk, NY, USA), and the graphics were generated in GraphPad Prism version 5 (GraphPad, La Jolla, CA, USA).

\section{RESULTS}

The baseline characteristics of 83 RA patients and 12 healthy controls were listed in Table 1. The concentrations of IL-33 and IL-6 were significantly higher in patients with RA than in controls $(p<0.001$ for both; Figure 1). The median IL-33 level for RA patients was $18.37 \mathrm{pg} / \mathrm{mL}$ (range, 0.0-4364.2 pg/mL), whereas that for the controls was $0 \mathrm{pg} / \mathrm{mL}$ (range, 0.0-15.97 pg/mL). The median IL-6 levels were $8.4 \mathrm{pg} / \mathrm{mL}$ (range, $0.6-20 \mathrm{pg} / \mathrm{mL}$ ) and $1.25 \mathrm{pg} / \mathrm{mL}$ (range, $0.3-2.9 \mathrm{pg} / \mathrm{mL}$ ), respectively.
We examined the relationship between serum IL-33 or IL-6 levels and RA characteristics at baseline in all 83 RA patients. IL-33 levels showed a significant correlation with RF titer (Spearman's rank correlation coefficient $[\mathrm{rho}=0.660, \mathrm{p}<0.001)$, but not with DAS28 (rho=0.122, $\mathrm{p}=0.271), \quad \mathrm{ESR} \quad$ (rho $=0.177$, $\mathrm{p}=0.110)$, hsCRP (rho=0.151, $\mathrm{p}=0.174)$, or RA duration (rho=0.121, $\mathrm{p}=0.274)$. IL-6 levels showed a significant correlation with hsCRP (rho $=0.482, \mathrm{p}<0.001)$, but not with DAS28 (rho $=0.204, p=0.065)$, ESR ( $\mathrm{rho}=0.104$, $\mathrm{p}=0.351)$, RF titer ( $\mathrm{rho}=-0.028, \mathrm{p}=0.805)$, or $\mathrm{RA}$ duration ( $\mathrm{rho}=-0.170, \mathrm{p}=0.124$ ). There was no correlation between serum IL-6 and IL-33 levels ( $\mathrm{rho}=0.012, \mathrm{p}=0.913$ ).

We compared serum IL-33 levels in 40 RA patients before and after tocilizumab therapy. The results showed that serum IL-33 levels decreased significantly after 24 weeks of tocilizumab (median $77.0 \mathrm{pg} / \mathrm{mL}$ down to median $11.9 \mathrm{pg} / \mathrm{mL}$, $\mathrm{p}<0.001$; Figure 2a). There was no difference in serum IL-6 levels at baseline and those after 24 weeks of tocilizumab therapy (median 7.1 and $8.9 \mathrm{pg} / \mathrm{mL}$, respectively, $\mathrm{p}=0.503$; Figure 2b). Serum IL-33 levels in ACR20 responders decreased significantly after 24 weeks of tocilizumab therapy (median 109.0 to median $15.8 \mathrm{pg} / \mathrm{mL}$, 
$\mathrm{n}=29 ; \mathrm{p}<0.001$ ); this decrease was not significant in non-responders (median 35.5 to $12.1 \mathrm{pg} / \mathrm{mL}$, $\mathrm{n}=11, \mathrm{p}=0.084)$. Changes in IL-6 levels in ACR20 responders (6.9 to $7.2 \mathrm{pg} / \mathrm{mL}, \mathrm{p}=0.477)$ and nonresponders ( 8.7 to $10.6 \mathrm{pg} / \mathrm{mL}, \mathrm{p}=0.665$ ) between baseline and 24 weeks after tocilizumab therapy were not significant. Baseline IL-33 levels in the two subgroups were not significantly different $(p=0.765$; Figure 3a). The same was true for IL-6 $(p=0.267$; Figure $3 b)$.

\section{DISCUSSION}

Serum IL-33 and IL- 6 concentrations in patients with RA were significantly higher than those in healthy controls. The significant correlation between IL-33 and RF titer suggests that production of the former might be linked to autoantibodies in these patients. IL-33 enhances mast cell activation by immunoglobulin $\mathrm{G},{ }^{12}$ as well as inducing human mast cells to release immunomodulatory mediators such as IL-10 and histamine along with other cytokines associated with type 2 immune responses, such as IL-5 and IL-13. IL-33 primes mast cells toward a Th2/immunomodulatory phenotype, a phenotype that becomes more prominent after concomitant activation of mast cells by immunoglobulin G. ${ }^{12}$

Like the majority of IL-1 family members that act as pro-inflammatory cytokines, IL-33 is expected to promote inflammation in RA. ${ }^{13}$ Studies of mice with CIA suggest that inhibiting the IL-33 receptor may have therapeutic effects similar to IL-1 inhibition therapy for RA. ${ }^{14}$ However, we found no correlation between the IL-33 concentrations and markers of RA disease activity (i.e., the DAS28, ESR, or hsCRP). The link between IL-33 and inflammation in RA may be indirect, and IL-33 levels could be affected by other cytokine cascades such as TNF- $\alpha$ or IL- 6 . TNF- $\alpha$ stimulates production of IL-33 by synovial fibroblasts, ${ }^{15}$ and IL-33 levels decrease after infliximab and etanercept therapy. ${ }^{16,17}$ In addition, overexpression of IL-33 increases TNF- $\alpha$-induced IL- 6 secretion by RA-synovial fibroblasts, and IL-33 silencing significantly down-regulates TNF- $\alpha$-induced IL- 6 secretion in vitro. ${ }^{10}$ However, we detected no direct correlation between serum IL-6 and IL-33 levels in this study. We did find that IL-33 levels showed a high degree of inter-individual variability, which may (at least in part) account for discrepancies regarding the correlation between serum IL-33 concentrations and RA disease activity. ${ }^{16-18}$

There are several limitations in this study. Because only 40 patients received tocilizumab therapy and the majority showed a favorable response to tocilizumab therapy, the sample size of non-responders was too small $(n=11)$. The sample size of healthy controls was also too small to provide a precise match in age and sex with RA patients.

However, to the best of our knowledge, this study is the first to examine serum IL-33 concentrations both before and after tocilizumab therapy. Although the sample size was small, all patients received a stable dose of methotrexate and/or other disease-modifying anti-rheumatic drugs, and we observed a relatively long term response after 24 weeks of therapy. Unfortunately, we did not analyze radiologic progression and therefore cannot present results showing a correlation between IL-33 and joint damage. This is another limitation of this study.

In conclusion, our results revealed that serum levels of IL-33 did not correlate with IL- 6 levels. In addition, treatment with an IL-6R inhibitor did not change serum IL-6 levels; rather, it reduced serum IL-33 levels. It is interesting that even in ACR20 responders with decreased ESR and CRP levels, the pro-inflammatory cytokine IL-6 level did not change significantly in the present study. There was a previous report showing that serum IL- 6 concentrations are increased after administration of tocilizumab in both healthy volunteers and RA patients and suggesting that free serum IL-6 is increased after tocilizumab therapy because IL-6R-mediated consumption of IL- 6 was inhibited by the unavailability of tocilizumab-free IL-6R. ${ }^{19}$ Taken together, it is not possible at the present time to explain the response to tocilizumab therapy in terms of serum IL- 6 levels; therefore, IL-33 may be a useful indicator of responses to tocilizumab therapy.

\section{Declaration of conflicting interests}

The authors declared no conflicts of interest with respect to the authorship and/or publication of this article. 


\section{Funding}

This research was supported by grants from the Ministry of Science, ICT and Future Planning, Republic of Korea (NRF-2015M3A9B6052011) and the Korea Healthcare technology R\&D Project, Ministry of Health and Welfare, Republic of Korea (HI14C1277), and partly by a grant from JW Pharmaceuticals.

\section{REFERENCES}

1. McInnes IB, Schett G. Cytokines in the pathogenesis of rheumatoid arthritis. Nat Rev Immunol 2007;7:429-42.

2. Ulfgren AK, Lindblad S, Klareskog L, Andersson J, Andersson U. Detection of cytokine producing cells in the synovial membrane from patients with rheumatoid arthritis. Ann Rheum Dis 1995;54:654-61.

3. Maini R, St Clair EW, Breedveld F, Furst D, Kalden $\mathrm{J}$, Weisman $\mathrm{M}$, et al. Infliximab (chimeric antitumour necrosis factor alpha monoclonal antibody) versus placebo in rheumatoid arthritis patients receiving concomitant methotrexate: a randomised phase III trial. ATTRACT Study Group. Lancet 1999;354:1932-9.

4. Cohen SB, Moreland LW, Cush JJ, Greenwald MW, Block S, Shergy WJ, et al. A multicentre, double blind, randomised, placebo controlled trial of anakinra (Kineret), a recombinant interleukin 1 receptor antagonist, in patients with rheumatoid arthritis treated with background methotrexate. Ann Rheum Dis 2004;63:1062-8.

5. Fleischmann RM, Halland AM, Brzosko M, BurgosVargas R, Mela C, Vernon E, et al. Tocilizumab inhibits structural joint damage and improves physical function in patients with rheumatoid arthritis and inadequate responses to methotrexate: LITHE study 2-year results. J Rheumatol 2013;40:113-26.

6. Liew FY, Pitman NI, McInnes IB. Disease-associated functions of IL-33: the new kid in the IL-1 family. Nat Rev Immunol 2010;10:103-10.

7. Miller AM. Role of IL-33 in inflammation and disease. $\mathrm{J}$ Inflamm (Lond) 2011;8:22.

8. Hong YS, Moon SJ, Joo YB, Jeon $\mathrm{CH}$, Cho $\mathrm{ML}$, Ju JH, et al. Measurement of interleukin-33 (IL-33) and IL-33 receptors (sST2 and ST2L) in patients with rheumatoid arthritis. J Korean Med Sci 2011;26:1132-9.
9. Talabot-Ayer D, McKee T, Gindre P, Bas S, Baeten DL, Gabay C, et al. Distinct serum and synovial fluid interleukin (IL)-33 levels in rheumatoid arthritis, psoriatic arthritis and osteoarthritis. Joint Bone Spine 2012;79:32-7.

10. Leung BP, Xu D, Culshaw S, McInnes IB, Liew FY. A novel therapy of murine collagen-induced arthritis with soluble T1/ST2. J Immunol 2004;173:145-50.

11. Palmer G, Talabot-Ayer D, Lamacchia C, Toy $\mathrm{D}$, Seemayer CA, Viatte $\mathrm{S}$, et al. Inhibition of interleukin-33 signaling attenuates the severity of experimental arthritis. Arthritis Rheum 2009;60:738-49.

12. Rivellese F, Suurmond J, Habets K, Dorjée AL, Ramamoorthi N, Townsend MJ, et al. Ability of interleukin-33- and immune complex-triggered activation of human mast cells to down-regulate monocyte-mediated immune responses. Arthritis Rheumatol 2015;67:2343-53.

13. Barksby HE, Lea SR, Preshaw PM, Taylor JJ. The expanding family of interleukin-1 cytokines and their role in destructive inflammatory disorders. Clin Exp Immunol 2007;149:217-25.

14. Zhao Q, Chen G. Role of IL-33 and its receptor in T cell-mediated autoimmune diseases. Biomed Res Int 2014;2014:587376.

15. Kunisch E, Chakilam S, Gandesiri M, Kinne RW. IL-33 regulates TNF- $\alpha$ dependent effects in synovial fibroblasts. Int J Mol Med 2012;29:530-40.

16. Mu R, Huang HQ, Li YH, Li C, Ye H, Li ZG. Elevated serum interleukin 33 is associated with autoantibody production in patients with rheumatoid arthritis. J Rheumatol 2010;37:2006-13.

17. Kageyama Y, Torikai E, Tsujimura K, Kobayashi M. Involvement of IL-33 in the pathogenesis of rheumatoid arthritis: the effect of etanercept on the serum levels of IL-33. Mod Rheumatol 2012;22:89-93.

18. Tang S, Huang H, Hu F, Zhou W, Guo J, Jiang $\mathrm{H}$, et al. Increased IL-33 in synovial fluid and paired serum is associated with disease activity and autoantibodies in rheumatoid arthritis. Clin Dev Immunol 2013;2013:985301.

19. Nishimoto $\mathrm{N}$, Terao $\mathrm{K}$, Mima $\mathrm{T}$, Nakahara $\mathrm{H}$, Takagi N, Kakehi T. Mechanisms and pathologic significances in increase in serum interleukin-6 (IL-6) and soluble IL-6 receptor after administration of an anti-IL-6 receptor antibody, tocilizumab, in patients with rheumatoid arthritis and Castleman disease. Blood 2008;112:3959-64. 